\title{
Everyday Physical Activity and Sedentary Behavior After Total Joint Arthroplasty: Do Patients and Partners Develop an Active Lifestyle?
}

This article was published in the following Dove Press journal: Clinical Interventions in Aging

\author{
Tobias Kalisch (D) \\ Frank Horst ${ }^{2}$ \\ Georg Gosheger' \\ Christoph Theil (D) \\ Leonie Seeber' \\ Burkhard Moellenbeck' \\ 'Department of Orthopedics and Tumor \\ Orthopedics, Muenster University \\ Hospital, Muenster, 48I49, Germany: \\ ${ }^{2}$ Department of Orthopedics and \\ Traumatology, St. Josef-Stift Sendenhorst, \\ Sendenhorst, 48324, Germany
}

Background: Osteoarthritis (OA) is a frequent cause of pain and disability, affecting half of the world's population aged 65 years or older. Due to experienced pain and limitations, OA patients generally spend most of their waking hours sedentary and fail to adhere to physical activity (PA) recommendations. As a result of living together with OA patients, their partners might adopt a sedentary lifestyle. This study investigates the change in habitual PA and sedentary behavior (SB) in patients and their partners one year after the patients' total joint replacement (arthroplasty) and inpatient rehabilitation.

Methods: This is a follow-up of a prospective cohort study including 24 older couples (69.3 \pm 7.8 years, gender ratio $11: 13$ ) consisting of OA patients (17 hip OA, 7 knee OA) and their partners. The participants' habitual PA and SB were assessed by means of synchronous accelerometry (16h per day, 4-7 days) and additional self-report.

Results: Although the partners ( $\sim 6300$ steps per day) were significantly more active than the patients ( 4800 steps per day) before arthroplasty, their lifestyle was still classified "low active". Irrespective of the method used, the PA and SB parameters of patients and partners were significantly correlated before arthroplasty (accelerometry: $r \geq 0.333, p \leq 0.001$; selfreport: $\mathrm{r} \geq 0.569, \mathrm{p} \leq 0.004$ ). Following the patients' arthroplasty, no improvement in PA or SB was observed either in the patients or their partners. A detailed evaluation of accelerometric data on hourly basis revealed no change in behavior for both groups. Furthermore, their everyday activities remained correlated (accelerometry: $r \geq 0.418, p \leq 0.001$; questionnaire: $\mathrm{r} \geq 0.554, \mathrm{p} \leq 0.005$ ).

Conclusion: One year post-arthroplasty and inpatient rehabilitation neither the OA patients nor their partners had changed their PA or SB. Their everyday physical activity was still strongly intertwined. Further research is needed to determine why behavior change does not occur even though the prerequisites are in place.

Keywords: osteoarthritis, dyads, physical activity, sedentary behavior, arthroplasty

\section{Plain Language Summary}

Osteoarthritis of the lower extremities joints is one of the most common diseases in the elderly. As a result of pain and restricted mobility, affected patients become inactive over time and finally spend most of the day sitting. However, this inactive lifestyle poses a great risk to their own health as other diseases, such as those of the cardiovascular system, can develop. Furthermore, it has been shown that the partners who live with these patients adopt the inactive lifestyle and thus unconsciously become a risk group too. Here we have investigated whether the implantation of an artificial joint leads to an improvement in everyday physical activity among patients and their partners. They were examined before
Department of Orthopedics and Tumor Orthopedics, Muenster University Hospital, Albert-Schweitzer-Campus I, Muenster, 48I49, NRW, Germany Tel +492518347984

Email tobias.kalisch@ukmuenster.de 
the patients' surgery and about one year after by means of questionnaires and body-worn motion sensors. Finally, we could not detect any change in the participating couples. Their everyday lifestyle was still characterized by low activity. This result means that the surgery and rehabilitation relieve patients of pain and enables them to move again - but the necessary behavioral changes do not occur. As a consequence, we propose the development of novel rehabilitation programs that actively involve both, patients and partners.

\section{Introduction}

Hip and knee Osteoarthritis (OA) is associated with decreased functional ability, poor quality of life, decreased physical activity (PA) as well as increased sedentary behavior (SB). ${ }^{1}$ Based on studies that have objectively examined the activity of OA patients under everyday conditions, the majority of patients are physically inactive and spend two-thirds of the day sitting. ${ }^{1,2}$ As a consequence, these patients do not accumulate enough moderate-to-vigorous PA (MVPA) to meet general guidelines for PA of at least 150 minutes MVPA per week. ${ }^{3}$ In fact, only about $13 \%$ percent of adults with hip or knee OA reach these generally recommended activity levels. ${ }^{1}$ Even the more moderate guidelines for people with OA (ie, 45 min of MVPA per week or at least 6000 steps per day) are not reached by the majority of patients. ${ }^{4-6}$ Since PA is a key element in the conventional treatment of $\mathrm{OA}$, it is reasonably that many patients opt for surgical treatment sooner or later. ${ }^{7}$ The most effective and efficient invasive treatment for $\mathrm{OA}$ is an arthroplasty which restores joint functionality and usually relieves the patient from pain as far as possible. ${ }^{8}$ However, contrary to the implicit expectations of many patients, the surgical joint replacement does not automatically lead to an increase in PA. ${ }^{9}$ The relevant literature is ambiguous for both knee and hip arthroplasty, as even six to twelve months after surgery only minor changes in PA were observed in patients. ${ }^{10-12}$

As with other chronic diseases, the partners of OA patients play an important role in the day-to-day management of disease-related symptoms and limitations by acting as informal caregivers. ${ }^{13}$ The specific interdependencies and mutual influences of OA patients and their partners have only been investigated sporadically and several potential relevant factors remain unknown. ${ }^{14}$ However, living with OA not only affects the patients, but also their cohabitating partners, altering individual roles and responsibilities within the couples. ${ }^{15}$ Even though informal caregiving can facilitate a positive outcome for
OA patients following arthroplasty, little is known about the situation of caring partners themselves. ${ }^{16,17}$ Actually, these partners experience lower levels of psychological well-being, as well as physical burdens indicating that it is a challenge for them to support their partners' recovery. ${ }^{18,19}$ There are various interdependencies between patients and their partners regarding mental and physical health as well as health behaviour. ${ }^{20}$ Consequently, it is likely that the inactive lifestyle of OA patients might be adopted by their partners. Indeed, it has recently been shown that OA patient-partner dyads differ significantly from control couples in terms of $\mathrm{PA} .{ }^{21}$ With regard to SB, it was even found that both partners and controls were in fact at the same level as the OA patients, which could be attributed to the widespread sedentary lifestyle in today's society. $^{22,23}$

Behavioral alignment between OA patients and their partners may generally limit the supporting role of partners and needs to be further investigated. ${ }^{21}$ Therefore, the primary objective of this study was to objectively assess long-term changes of PA in OA patients and their partners following arthroplasty and inpatient rehabilitation. Our hypothesis stated that current measures are not sufficient to alter the couples' health-related behavior and therefore the investigated parameters should remain unchanged.

\section{Materials and Methods}

This follow-up of a prospective cohort study analyzed longitudinal accelerometer data from a group of older OA patients undergoing primary elective THA or TKA and their partners. Institutional review board (WWU Muenster, Germany) approval was obtained before the initiation of the study. Data were collected from April 2018 to September 2019. The trial has been registered with the German Clinical Trials Register (DRKS00014292). Details of the study protocol have been previously described. ${ }^{21}$

All participants were recruited in high-volume hospitals specialized in total joint replacements (Sendenhorst and Muenster, Germany). Inclusion criteria for the study were German-speaking patients aged between 50 and 85 years, who met the American Rheumatology Association criteria for the classification of hip or knee OA and were refractory to conservative medical therapy before arthroplasty. $^{24}$ Patients undergoing revision arthroplasty procedures were excluded. Furthermore, they should not have any other help in everyday life than their cohabitating partners (eg, skilled nursing). Partners were self-identified 
by the patient and could include any person regardless of legal status of their relationship as long as they were cohabitating at the time of inclusion. The exclusion criteria for all participants were defined as any medical condition or medication significantly restricting PA and mobility. More than four co-morbidities (out of ten investigated conditions, see Table 1) led to exclusion from the study. Both the patients and their partners had to be able to move under everyday conditions without assistive devices. Baseline observations were done two to three weeks prior to the patients' arthroplasty. All patients underwent conventional arthroplasty by a senior orthopaedic surgeon and received standardized postoperative care including appropriate analgesia and mechanical prophylaxis against venous thromboembolic events. Ambulation began on the day of surgery. Within one week after arthroplasty, the patients were discharged to the same inpatient rehabilitation facility (Sendenhorst, Germany) to participate in a structured rehabilitation program. After an average stay of three to four weeks, they were finally discharged to their homes. Patients and partners were followed up for one year after surgery. All investigations were performed by a single examiner and data was collected from patients and partners at the same time. All subjects provided written informed consent.

A general health questionnaire was used to determine the participants' eligibility for the study by obtaining information on health history, history of OA, medication, alcohol consumption, smoking, previous or scheduled surgery and present or absence of any co-morbidities. In detail, ten co-morbidities (cardiovascular, pulmonary, metabolic, gastrointestinal, of the liver, kidney or blood as well as cancer, depression and musculoskeletal diseases) that were currently being treated by a physician or for which medication was regularly taken were recorded.

\section{Primary Outcome Measures}

PA and SB were objectively measured with the ActiGraph wGT3X-BT accelerometer (v1.9.2, ActiGraph, LLC, Fort Walton Beach, FL, USA). The accelerometer is a light and small device capable of recording accelerations in three axes as well as inclination. It was attached to an elastic belt that study participants wore around the waist on the dominant body side (according to handedness). Data were recorded with a sampling rate of $100 \mathrm{~Hz}$ in response to the magnitude of $\pm 8 \mathrm{~g}$ and integrated over an epoch length of

Table I Characteristics Among OA Patients and Their Partners

\begin{tabular}{|c|c|c|c|}
\hline Characteristics & OA Patients (n or M \pm SD) & Partners ( $n$ or $M \pm S D$ ) & $\begin{array}{l}\text { Difference Between Groups ( } t \text {-Test/Chi- } \\
\text { Square) }\end{array}$ \\
\hline $\begin{array}{l}\text { Time between } \\
\text { assessments }\end{array}$ & \multicolumn{2}{|c|}{$11.8 \pm 1.3$ months (range II-16 months) } & - \\
\hline \multicolumn{4}{|l|}{ Demographics } \\
\hline Age & $69.4 \pm 7.2$ (range $52-81$ years) & $69.1 \pm 8.6$ (range $50-83$ years) & $p=0.885$ \\
\hline Gender & II male, I3 female & I3 male, II female & $\chi^{2}=0.333 ; p=0.564$ \\
\hline $\mathrm{BMI}^{\mathrm{a}}$ & $26.6 \pm 3.8$ & $27.0 \pm 4.1$ & $p=0.775$ \\
\hline Education ${ }^{\mathrm{b}}$ & $6 \mathrm{sll}, 13 \mathrm{sl} 2,\left.3 \mathrm{tt}\right|^{\S}$ & $10 \mathrm{sl}, \mathrm{II} \mathrm{s}|2,2 \mathrm{t}|^{\S}$ & $\chi^{2}=1.345 ; p=0.510$ \\
\hline Occupation $^{c}$ & $19 \mathrm{r}, 3 \mathrm{pt}, 2 \mathrm{ft}$ & $18 \mathrm{r}, 2 \mathrm{pt}, 4 \mathrm{ft}$ & $\chi^{2}=0.894 ; p=0.640$ \\
\hline Comorbidities $^{d}$ & $1.3 \pm 0.8($ range $0-3)$ & $1.1 \pm 0.9($ range $0-3)$ & $p=0.397$ \\
\hline \multicolumn{4}{|l|}{ Perioperative details } \\
\hline Affected joint & I7 hip, 7 knee & - & - \\
\hline Initial diagnosis of $\mathrm{OA}$ & $6.9 \pm 6.6($ range $0.5-28)$ & - & - \\
\hline Pain medication ${ }^{\mathrm{e}}$ & $4 \mathrm{n}, 10 \mathrm{i}, 2 \mathrm{w}, 3 \mathrm{ww}, 5 \mathrm{~d}$ & - & - \\
\hline
\end{tabular}

Notes: ${ }^{\mathrm{a} B o d y}$ mass index (body mass divided by the square of the body height $\left[\mathrm{kg} / \mathrm{m}^{2}\right]$ ). ${ }^{\mathrm{b}}$ Education levels: s/I (secondary level I; age: $10-15 / \mathrm{I} 6$ years), s/2 (secondary level II; age: $15-19$ years), $\mathrm{tl}$ (tertiary level; age: $>19$ years). ${ }^{\S}$ Data missing. ${ }^{\mathrm{C}}$ Occupation $\left(\mathrm{r}=\right.$ retired; $\mathrm{pt}=$ part time/occasional job; ft $=$ full time job). ${ }^{\mathrm{d}} \mathrm{Pathological}$ conditions (cardiovascular, pulmonary, metabolic, gastrointestinal, liver, kidney, blood, cancer, depression, musculoskeletal diseases) $(0=$ best condition; $10=$ worst condition).

${ }^{\mathrm{e} A n a l g e s i c}$ consumption related to osteoarthritis $(\mathrm{n}=$ none $|\mathrm{i}=\operatorname{irregular}| \mathrm{w}=$ weekly $\mid \mathrm{w} w=$ several times a week $\mid \mathrm{d}=$ daily). 
$10 \mathrm{~s}$ as activity counts. In a last step, these counts were translated into physiological meaningful indicators and presented as different PA/SB behaviors based on the calculated counts per minute (cpm). ${ }^{25}$ Patients and partners were instructed to wear the devices simultaneously. At night and while showering, bathing or swimming they had to remove the devices. The monitors were initialized as per the manufacturer's manual. Data were downloaded using the ActiLife Software (v6.13.3). The wear time was set to 10-16 hours per day for at least four days as these are minimally needed to obtain reliable $\mathrm{PA}$ and SB estimates. ${ }^{4}$ Non-wear times were automatically detected, cross-checked and excluded from analyses. ${ }^{26}$ Based on previous knowledge on this cohort, night hours (11:00 p.m. - 07:00 a.m.) were excluded by default as the participants typically slept during this time. The participants' SB was quantified by the total time in sedentary bouts and the average length of sedentary bouts. Their PA was quantified by the number of steps and the total time in MVPA. Common intercepts for uniaxial data analysis were used to classify sedentary bouts $(<150 \mathrm{cpm}$ for 15 minutes with a drop time of one minute) and common intercepts for triaxial data analysis were used to classify the time in MVPA (2690-6166 cpm). ${ }^{27,28}$ Since OA patients are known to execute their daily activity during a small portion of the waking hours and spend the rest of the time sitting, a detailed evaluation of the participants' PA and SB was conducted on an hourly basis. ${ }^{29}$

\section{Secondary Outcome Measures}

The Lequesne index (LI) of severity for OA covers specific symptoms and physical functional disability in patients suffering from hip or knee OA. ${ }^{30}$ It aggregates symptoms and function, where the subscale pain is analyzed by five items, maximum distance walked by two items and activities of daily living by four items. The score ranges from 0 to 24 (maximum pain/disability) and is calculated as the sum of all item scores where 0 is no handicap, 1-4: a mild handicap, 5-7: a moderate handicap, 8-10: a severe handicap, 11-13: a very severe handicap, and 14 equals an extremely severe handicap.

For the individual (subjective) assessment of PA, the German "Habitual Physical Activity Questionnaire" was used. ${ }^{31}$ This survey instrument quantifies the habitual PA of adult subjects by means of three distinct indices for work ( 7 items, total score 7-35 points), leisure time activities (excluding sport, 3 items, total score $3-15$ points) and sport (4 items, total score $4-20$ points). The higher the total score in the 3 indices, the more intense is the selfperceived PA. Since the number of working participants in this study was very low due to their age, only the two indices for leisure time activities and sport were evaluated.

\section{Statistical Analysis}

Data were analyzed using SPSS (v26, SPSS Inc, Chicago, IL, USA). All couples with at least four valid days of ActiGraph data (per person) at baseline and 12 months after arthroplasty were included in the analyses. Patient characteristics (general health and socio-economic data) were reported using descriptive statistics (mean and standard deviation or median and interquartile range). Either t-tests or chi-square tests depending on the type of variable were used to examine differences between the patients and their partners.

The four accelerometric parameters (total time in sedentary bouts, average length of sedentary bouts, total time in MVPA, number of steps) were presented descriptively using means and $95 \%$ confidence intervals (M, 95\% $\mathrm{CI})$ or medians and interquartile ranges (Mdn, IQR) on hourly basis, averaged over all recorded days for patients, their partners and as couple-specific performance deviations ( $\Delta$, reported as absolute values) at baseline and at 12 months follow-up. First, an outlier correction was performed with these data. By using boxplot figures, the outlying hourly averages ( $>1.5 \mathrm{xIQR})$ were identified and removed. Cleared data were checked for normal distribution by means of the Shapiro-Wilk test and Q-Q-diagrams. Consequently, the accelerometric data were evaluated using parametric testing (t-tests, repeated measures ANOVA, post hoc tests, Pearson correlations) and nonparametric testing (Friedman-tests, Wilcoxon signed-rank test, Spearman correlations). Correlations were defined as very weak $(<0.19)$, weak $(0.2-0.39)$, moderate $(0.4-0.59)$, strong (0.6-0.79) or very strong $(\geq 0.8) .^{32}$ Effect sizes (Cohen's d/Spearman's r) were calculated and reported along the results.

\section{Results}

Of the 32 couples included in the initial study (for a flow-chart $\left.\mathrm{see}^{21}\right), 24(75 \%)$ could be recruited to participate again. Five couples were no longer interested in participating for various reasons, two couples stopped participating for general health reasons and one couple could not be contacted. Based on the subjects' reports there was no dropout due to problems with the arthroplasty. 


\section{Sample Characteristics}

Table 1 shows detailed characteristics of all 48 participants.

\section{Patient-Reported Outcomes}

The Lequesne index, decreased significantly from 10.25 (Mdn, IQR: 8.00-12.50) points before arthroplasty to 4.00 (Mdn, IQR: 1.13-6.38) after arthroplasty $(\mathrm{z}=-4.289, \mathrm{p} \leq$ $0.001)$ representing a strong effect $(r=0.875)$. This result describes a change from a joint-related "severe handicap" under everyday conditions to a "mild handicap". ${ }^{30}$

\section{The Habitual Physical Activity (HPA) Questionnaire}

The analysis of the patients' questionnaire data resulted in a leisure time activities (lta) index of $8.04 \pm 2.84$ and a sport (s) index of $9.54 \pm 2.81$ before arthroplasty. The corresponding indices calculated for their partners (lta: $8.50 \pm 2.57 ; s: 10.25 \pm 2.80)$ did not differ significantly (lta: $\mathrm{p}=0.560 ; s: \mathrm{p}=0.387$ ) and were strongly correlated with the patients' data (lta: $\mathrm{r}=0.569, \mathrm{p}=0.004 ; \mathrm{s}: \mathrm{r}=$ $0.588, \mathrm{p}=0.003)$. Following arthroplasty, the patients' questionnaire data (1ta: $8.42 \pm 2.60 ; s: 10.13 \pm 2.98)$ and their partners' data (lta: $8.67 \pm 2.97 ; s: 10.38 \pm 2.70$ ) remained unchanged compared to the previous situation (patients' lta: $\mathrm{p}=0.443$ and $s: \mathrm{p}=0.262$; their partners lta: $\mathrm{p}=0.676$ and $s: \mathrm{p}=0.752$ ). There were still no significant differences between the data both groups (lta: $\mathrm{p}=0.758 ; \mathrm{s}: \mathrm{p}=0.762)$ and the indices were still strongly correlated (lta: $\mathrm{r}=0.567, \mathrm{p}=0.004 ; \mathrm{s}: \mathrm{r}=$ $0.554, \mathrm{p}=0.005$ ). For both indices no arthroplastyrelated changes in the degree of correlation could be found (lta: $\mathrm{z}=0.010, \mathrm{p}=0.992 ; s: \mathrm{z}=0.164, \mathrm{p}=0.870$ ).

\section{Assessment of Habitual PA and SB by Accelerometry}

On average, the couples' activity was recorded on $6.0 \pm$ 0.8 days (range 5-7 days) thereof $2.0 \pm 0.7$ weekend days. Of the 24 data sets per group (ie, patients, partners and couple-specific deviations), each recorded over $16 \mathrm{~h}$ for the 4 parameters of accelerometry (ie, total of 1536 hourly means), 49 single values of the patients (3.2\%), 44 single values of the partners $(2.9 \%)$ and 71 single values $(4.6 \%)$ of the couple-specific deviations had to be excluded after checking for outliers.
Data collected by accelerometry for the SB and the PA of the patients and their partners can be found in Table 2 . Basically, it could be shown that the patients had a more distinctive SB (ie, longer, uninterrupted sedentary time per hour) and lower PA (ie, reduced number of steps and time in MVPA) than their partners. For 3 out of the 4 investigated parameters, significant differences between both groups could be revealed both before $(\mathrm{p} \leq 0.017)$ as well as after arthroplasty $(\mathrm{p} \leq 0.022)$. Only the prearthroplasty lengths of sedentary bouts $(p=0.094)$ and the postarthroplasty total time in sedentary bouts $(p=0.128)$ showed no significant differences between the groups.

There were only two significant changes in the group of patients from pre- to post-arthroplasty condition. The average total time in sedentary bouts decreased $(p=0.014)$ while the average length of sedentary bouts increased $(p=$ 0.012 ). In contrast, no changes were observed in the partners' data $(p>0.346)$. Subsequent multiple comparisons (Tukey-HSD post hoc tests) of the patients' according data did not reveal any changes. No single hour (7a.m. - 22 p.m.) could be determined at which the observed changes met the significance criteria $(p>0.999)$ (Figure 1).

For all accelerometric parameters a significant correlation between the patients' and their partners' data was found. This applied both to the data collected before arthroplasty $(r \geq 0.333, p<0.001)$ and after arthroplasty $(r \geq 0.418, p<0.001)$. The strongest correlations were found for the PA parameter number of steps $(r \geq 0.535, p<$ 0.001 , Figure 2).

The average couple-specific performance ratios were calculated as absolute hourly deviations between the patients' and their partners' SB as well as PA. No significant changes from pre- to postarthroplasty condition were found for the SB parameters total time in sedentary bouts and length of sedentary bouts $(p=0.205, p=0.750$; Table 3 and Figure $3 \mathrm{~A}$ and $\mathrm{B}$ ) and the two PA parameters number of steps and total time in MVPA ( $p=0.352, p=0.914$; Table 3 and Figure $3 \mathrm{C}$ and $\mathrm{D}$ ).

\section{Discussion}

In the current study, a combined assessment consisting of accelerometry and self-report was applied to OA patients and their cohabitating partners before and one year after the patients' arthroplasty to investigate behavioral changes in terms of habitual everyday activity. Findings showed that all analyzed aspects of the couples' PA and SB were significantly correlated before and after arthroplasty and 
Table 2 Physical Performance of OA Patients and Their Partners

\begin{tabular}{|c|c|c|c|c|}
\hline Physical Performance & Patients & Partners & Difference & $\begin{array}{c}\text { Correlation } \\
\text { Analysis }\end{array}$ \\
\hline $\begin{array}{l}\text { Total time in sedentary } \\
\text { bouts ( } \mathrm{min})\end{array}$ & \multicolumn{2}{|c|}{$M[95 \% \mathrm{Cl}]$} & & \\
\hline Pre-arthroplasty & $24.70[23.39,26.00]$ & $22.40[21.20,23.60]$ & $F_{(1,791)}=5.770, p=0.017^{*}$ & $r=0.502, p<0.001$ \\
\hline Post-arthroplasty & $24.30[23.08,25.52]$ & $22.82[21.60,24.03]$ & $F_{(1,796)}=2.328, p=0.128$ & $r=0.510, p<0.001$ \\
\hline Change & $\begin{array}{c}F_{(1,379)}=5.403, p=0.014^{*} \\
(d=0.029)\end{array}$ & $\begin{array}{c}F_{(1,374)}=7.018, p=0.744 \\
(d=-0.031)\end{array}$ & & $z=-0.147, p=0.883$ \\
\hline $\begin{array}{l}\text { Length of sedentary } \\
\text { bouts ( } \mathrm{min})\end{array}$ & \multicolumn{2}{|c|}{$\mathrm{M}[95 \% \mathrm{Cl}]$} & & \\
\hline Pre-arthroplasty & $14.95[14.20,|5.7|]$ & I4.04 [13.35, |4.73] & $F_{(I, 783)}=2.81 \mathrm{I}, \mathrm{P}=0.094$ & $r=0.354, p<0.001$ \\
\hline Post-arthroplasty & $15.20[14.42,15.97]$ & $13.86[13.15,14.56]$ & $F_{(1,778)}=5.283, p=0.022^{*}$ & $r=0.418, p<0.001$ \\
\hline Change & $\begin{array}{c}F_{(1,366)}=6.319, p=0.012^{*} \\
(d=-0.030)\end{array}$ & $\begin{array}{c}F_{(I, 362)}=0.89 I, p=0.346 \\
(d=0.025)\end{array}$ & & $z=-1.015, p=0.310$ \\
\hline Number of steps (\#) & \multicolumn{2}{|c|}{$\mathrm{M}[95 \% \mathrm{Cl}]$} & & \\
\hline Pre-arthroplasty & $300.06[280.29,319.84]$ & 395.57 [369.61, 421.52] & $\mathrm{F}_{(1,749)}=28.832, \mathrm{P}<0.00 \mathrm{I}^{*}$ & $r=0.630, p<0.001$ \\
\hline Post-arthroplasty & 319.29 [294.69, 343.89] & 366.19 [337.34, 395.04] & $F_{(1,770)}=5.336, P=0.02 I^{*}$ & $r=0.535, p<0.001$ \\
\hline Change & $\begin{array}{c}F_{(1,340)}=2.367, p=0.125 \\
(d=-0.090)\end{array}$ & $\begin{array}{c}F_{(1,349)}=0.395, p=0.530 \\
(d=0.110)\end{array}$ & & $z=1.898, p=0.058$ \\
\hline $\begin{array}{l}\text { Total time in MVPA } \\
(\mathrm{min})\end{array}$ & \multicolumn{2}{|c|}{$\mathrm{M}[95 \% \mathrm{Cl}]$} & & \\
\hline Pre-arthroplasty & $3.03[2.78,3.29]$ & $4.69[4.35,5.04]$ & $\mathrm{F}_{(1,780)}=54.325, \mathrm{p}<0.00 \mathrm{I}^{*}$ & $r=0.333, p<0.001$ \\
\hline Post-arthroplasty & $2.85[2.60,3.11]$ & $3.88[3.57,4.20]$ & $F_{(I, 752)}=24.055, p<0.001 *$ & $r=0.420, p<0.001$ \\
\hline Change & $\begin{array}{c}F_{(1,344)}=1.559, p=0.213 \\
(d=0.07 I)\end{array}$ & $\begin{array}{c}F_{(1,358)}=0.3 \mathrm{II}, \mathrm{p}=0.578 \\
(\mathrm{~d}=0.248)\end{array}$ & & $z=-1.342, p=0.180$ \\
\hline
\end{tabular}

Notes: The analysis of accelerometric data of the two groups was based on individual hourly marginal means (M) (I6h, 07:00 a.m. - II:00 p.m.). Changes were analyzed by means of repeated measures ANOVA, ANOVA and Fisher's z transformation of correlation coefficients. *Significant result $(p<0.05)$.

that no substantial activity changes had occurred regardless of whether the data were collected by self-report or accelerometry. Although the partners were somewhat more active than the patients at all times, both groups share a largely inactive lifestyle.

Martire et al have already been investigating how spouses might influence knee OA patients to increase their everyday PA. It was found that those spouses who were active themselves were able to influence the patients to significantly increase their PA. The authors concluded that interventions for knee OA should therefore target PA in both partners and use spousal strategies for helping patients to stay active. ${ }^{14}$ Contrasting the study mentioned above, the participants in our study are predominantly retired which promotes an alignment of their everyday behavior. ${ }^{33}$ In the first part of this study conducted in 2019 , we examined the deviation of daily activities in OAaffected couples and compared it to control couples. ${ }^{21} \mathrm{We}$ found that the deviations were lower in the target couples because the partners had obviously adapted to the patients' sedentary lifestyle. By analyzing the data on an hourly basis, we were able to show that the couples' PA was particularly synchronized in the morning and evening hours, which is in accordance with the current literature. Pauly and colleagues revealed that this synchronization is associated with more time spent together, longer relationship duration, and the perception of partner closeness. ${ }^{34}$ Based on these findings, two conclusions could be drawn: First, OA patients' partners may represent a previously little-noticed risk group for diseases resulting from a sedentary lifestyle. ${ }^{35}$ Secondly, these partners are unlikely to be able to fulfill their intended role of support after the patients' rehabilitation. Their adapted sedentary lifestyle may even impede a change in the patients' behavior as the mutual influence of cohabiting couples can result in both, positive and negative shared health behaviors. ${ }^{20}$ 

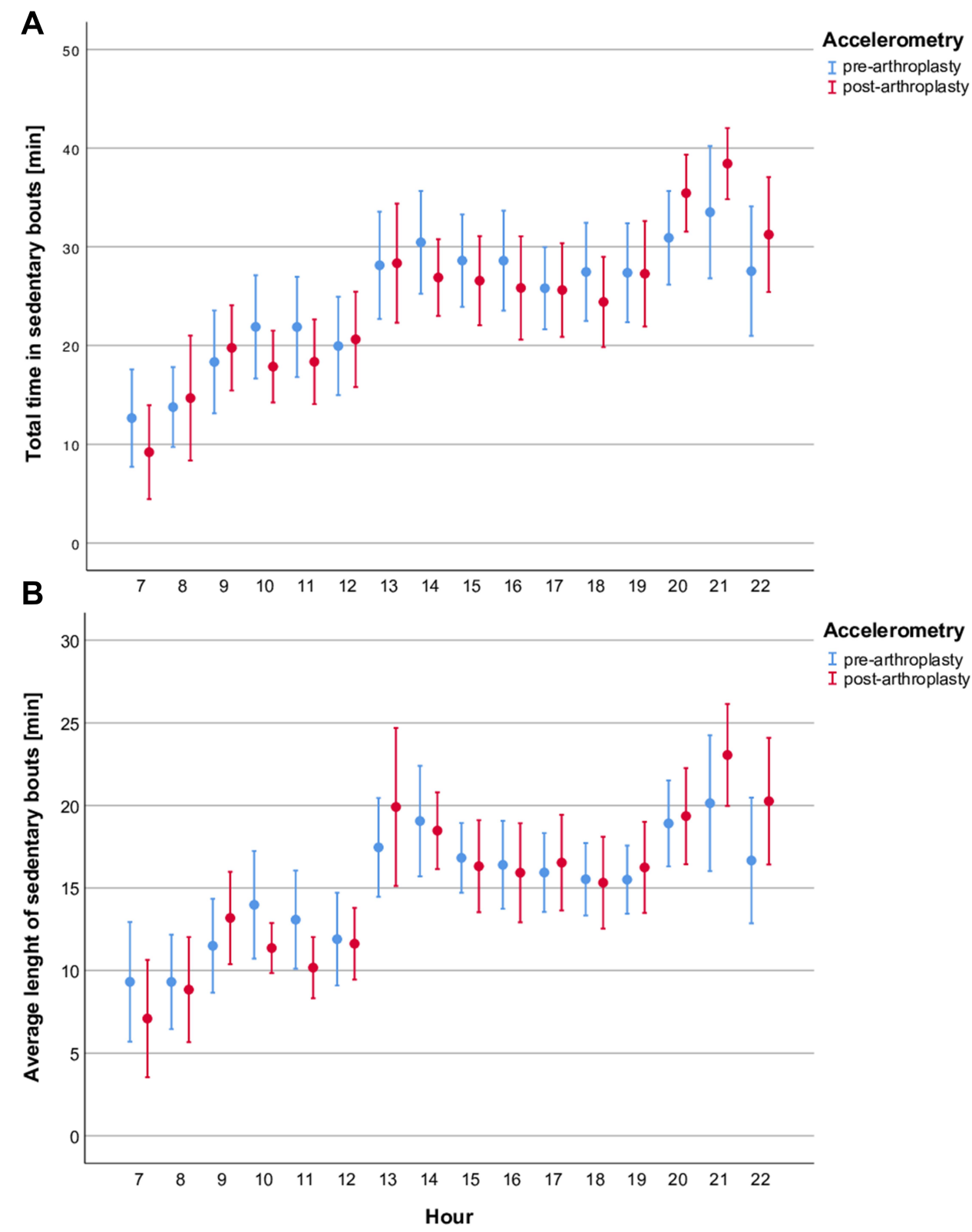

\section{Accelerometry}

I pre-arthroplasty

I post-arthroplasty

Figure I Daytime dependency of the patients' SB before and after arthroplasty. From pre-arthroplasty condition (blue coloring) to post-arthroplasty condition (red coloring), the patients' total time in sedentary bouts decreased $(\mathbf{A} ; p=0.014)$ while the average length of sedentary bouts increased $(\mathbf{B} ; p=0.012)$. The observed changes were distributed over the course of the day, with the result that no significant change of the investigated parameters could be determined for a single hour ( $p>0.999)$. The data are displayed as $\mathrm{M} \pm 95 \% \mathrm{Cl}$.

Recent studies on the outcome of joint arthroplasty in OA patients have included the patients' significant others (ie, spouses, family members, friends), as their personal situation is closely linked to the patients' condition and their perceptions might be of substantial value. ${ }^{36}$ Although it is generally known that cohabitating partners support OA patients in many ways and assume the role as informal caregiver there is still a paucity of research on caregiver burden in the setting of end-stage OA.
Specifically, there is little published literature evaluating the effects of total joint arthroplasty on patient's significant others. ${ }^{36}$ Kunkel et al used similar outcome metrics in hip and knee OA patients and their spouses before and repeatedly after arthroplasty. They found strong correlation between the patients' and their partners' mental status scores as well as moderate correlations for some physical function domains. ${ }^{36}$ Their findings suggested that partners closely share the patients' mental and even 


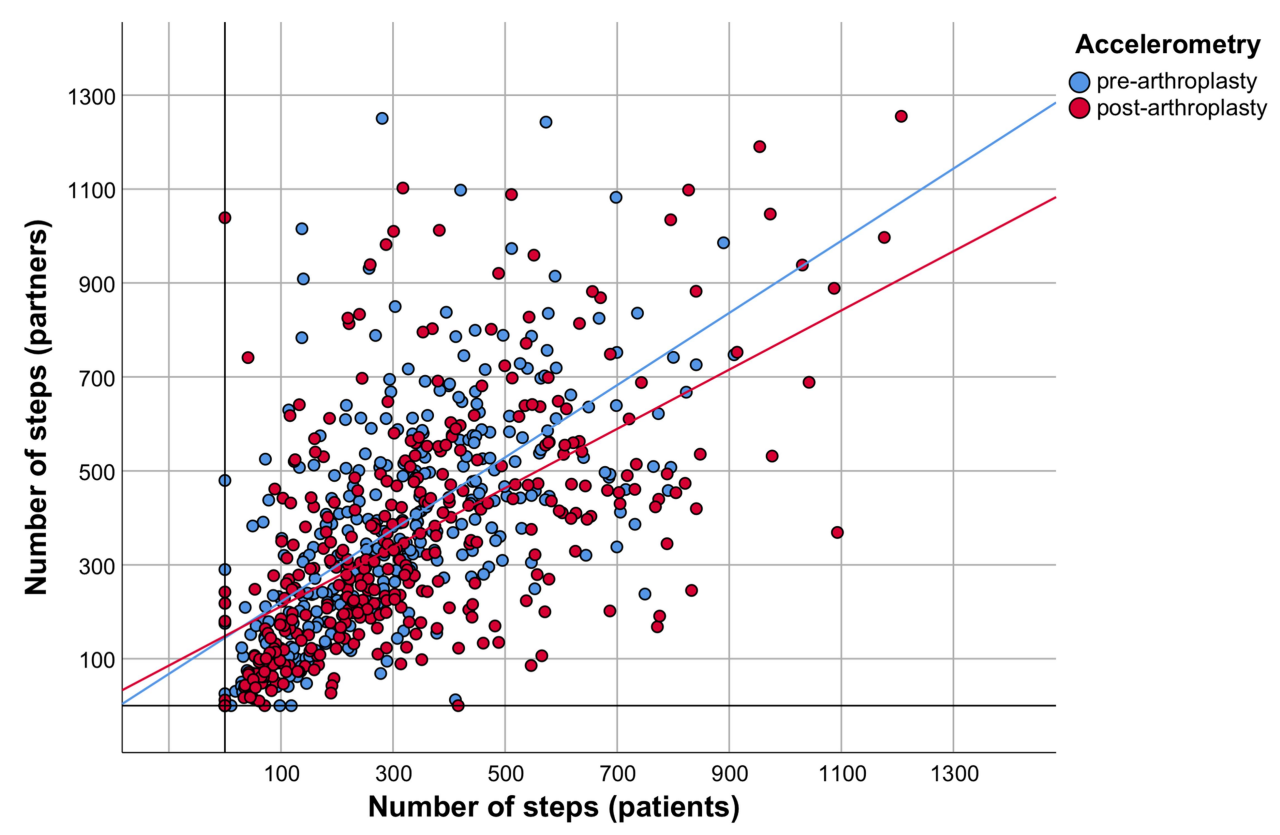

Figure 2 Correlation analysis of the patients' and their partners' PA. The correlation of hourly number of steps before arthroplasty (blue coloring, $r=0.630, p<0.00 \mathrm{I}$ ) and after arthroplasty (red coloring, $r=0.535, p<0.001)$ did not change significantly $(z=1.898, p=0.058)$.

some of the physical burden of disease and recovery. This has also been confirmed by other studies evaluating the condition of elderly couples affected by other chronic diseases. ${ }^{37}$ In a study on caregiver stress before and after hip arthroplasty, Chow et al showed that $91.3 \%$ of caregivers reported a level of stress ranging from "moderately stressful" to "very stressful" in connection with their caregiving role before the surgery, and $52 \%$ of caregivers still reported that they were "quite stressed" even three months after the operation. ${ }^{40}$ The results suggest that while arthroplasty is highly likely to eliminate pain and limitations in joint functionality, it does not automatically change the everyday life of OA-affected couples. These findings are in line with the data presented in this study and our previous work on the health-related quality of life in OA patients and their partners. ${ }^{19}$

By analyzing the PA of OA patients and partners on an hourly basis in the present study, we aimed to investigate whether and to what extent the degree of synchronization in behavior changes after surgery. If the patients had actually changed their PA, this would have been possible by an increase in everyday activity (steps) spread over the whole day, a concentrated activity increase (MVPA) in certain hours or a combination of both. According to our hypothesis, however, none of the cases occurred. The change in SB observed exclusively in patients does not represent an

Table 3 Couple-Specific Performance Ratios of Hourly PA and SB

\begin{tabular}{|c|c|c|c|c|}
\hline $\begin{array}{l}\text { Couple-Specific Performance } \\
\text { Ratios }\end{array}$ & Pre-arthroplasty & Post-arthroplasty & $\begin{array}{l}\text { Analysis of Change in } \\
\text { Performance Ratios }\end{array}$ & $\begin{array}{l}\text { Effect Size } \\
\text { (Cohen's d) }\end{array}$ \\
\hline Sedentary behavior & \multicolumn{2}{|c|}{ Mdn [IQR] } & & \\
\hline Total time in sedentary bouts ( $\Delta \mathrm{min})$ & $7.58[2.86,14.18]$ & $6.25[2.83,12.37]$ & $X_{F}^{2}(I)=1.609, p=0.205$ & 0.016 \\
\hline Length of sedentary bouts ( $\Delta \mathrm{min})$ & $4.89[1.81,8.70]$ & $4.68[2.16,12.86]$ & $X^{2}{ }_{F}(I)=0.102, p=0.750$ & 0.007 \\
\hline Physical activity & \multicolumn{2}{|c|}{ Mdn [IQR] } & & \\
\hline Number of steps $(\Delta \#)$ & $92.04[35.47,181.61]$ & $78.96[35.12,199.40]$ & $X^{2}{ }_{F}(I)=0.868, p=0.352$ & -0.114 \\
\hline Total time in MVPA ( $\Delta \mathrm{min})$ & $1.64[0.60,3.29]$ & $1.33[0.57,2.90]$ & $X^{2}{ }_{F}(I)=0.012, p=0.914$ & 0.066 \\
\hline
\end{tabular}

Notes: The four accelerometric parameters recorded in the patients and their partners were transformed into couple-specific hourly performance ratios $(\Delta$, ie, absolute deviations). Data are presented as medians (Mdn) and interquartile ranges (IQR). There were no significant changes from pre-arthroplasty to post-arthroplasty condition (P $\geq 0.205$ ). 
A

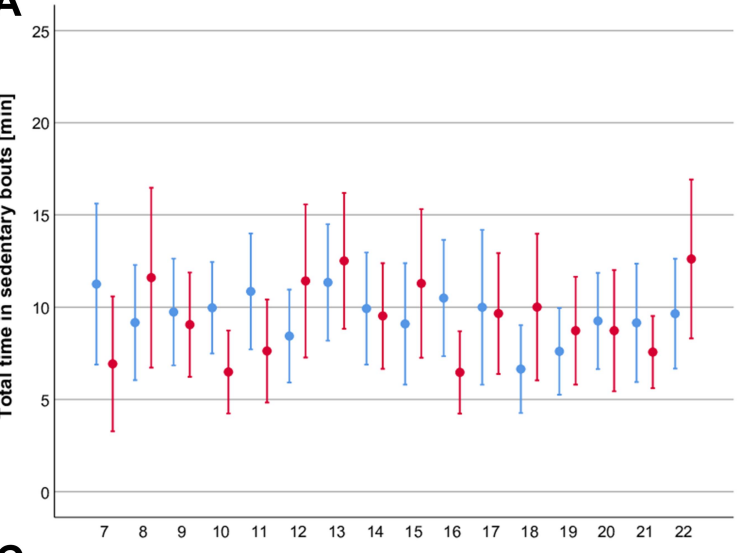

C

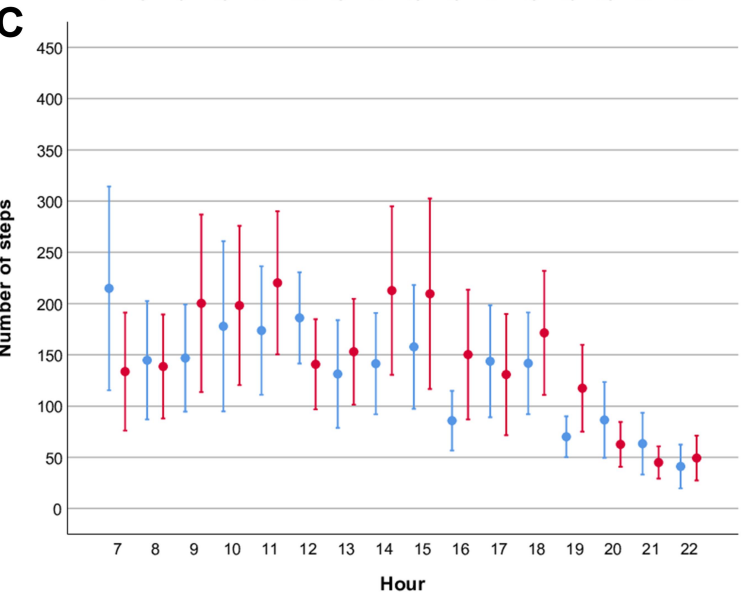

B

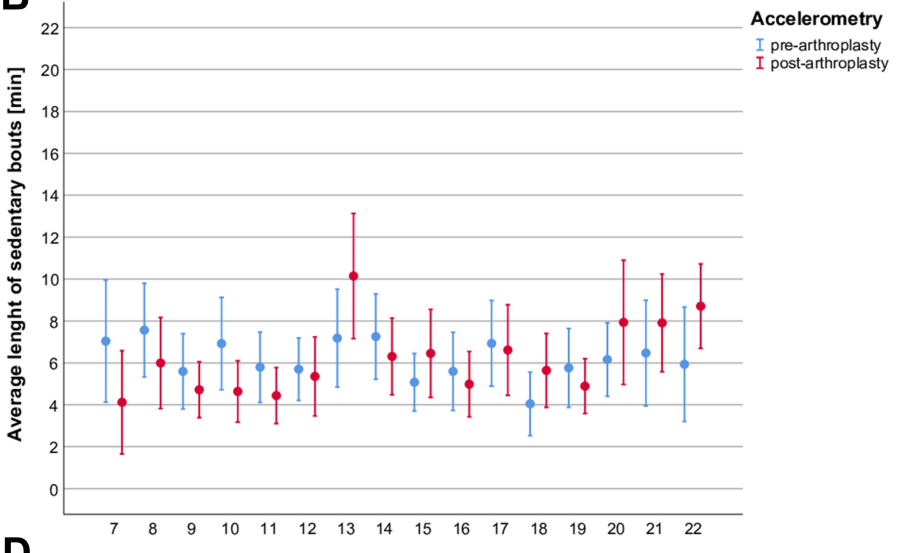

D

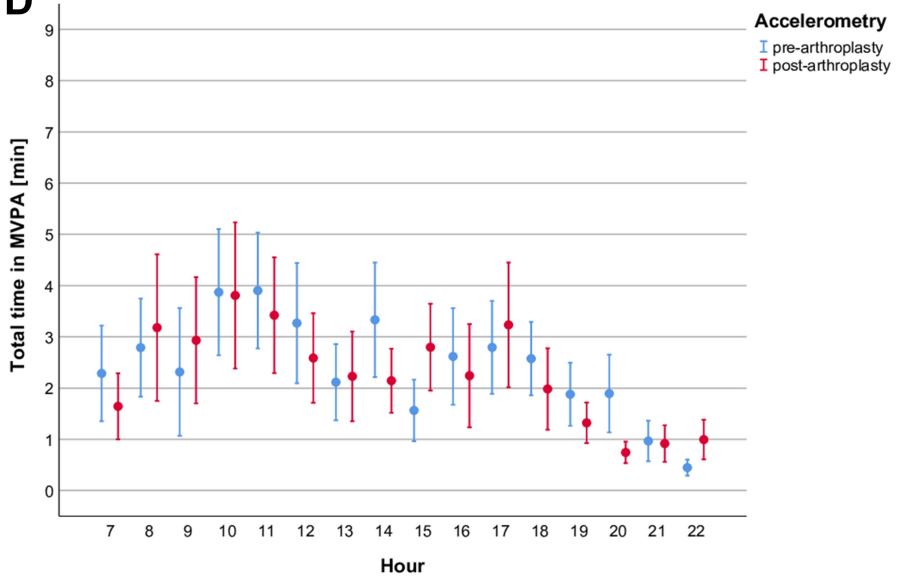

Figure 3 Hourly ratios of the couples' PA and SB. The hourly deviations of physical performance between the OA patients and their partners were calculated for the accelerometric parameters of SB (A: total time in sedentary bouts, B: average length of sedentary bouts) and PA (C: number of steps, D: total time in MVPA). There were no significant changes from pre-arthroplasty condition (blue coloring) to post-arthroplasty condition (red coloring) $(p \geq 0.205)$.

improvement either, since although the hourly total time spent sitting decreased, the average length of uninterrupted sitting increased. Furthermore, the observed effects are minimal. These findings are also consistent with previous investigations of different aspects of SB in this patient population. ${ }^{22}$

An unexpected result of the current study was the agreement between the questionnaire data and the accelerometric data in both groups. Usually, larger discrepancies between objective and subjective methods can be found, as it is a known problem that questionnaires on habitual PA may be open to bias and inaccuracy especially in older subjects. For this reason, the acquisition of PA in these subjects should be carried out using objective tests. ${ }^{38,39}$ Furthermore, the strong correlation between the patients' and partners' questionnaire data was remarkable. The type of query in the HPA questionnaire (ie, classification of activity, weekly time commitment and the total time in which the activity has already been performed in the last year) seems to be suitable for the patient-partner dyads studied here. The high importance of an objective description of PA in OA patients was recently emphasized by Sliepen and colleagues. Ideally, the FITT dimensions (frequency, intensity, time and type) of activities should be described to capture the individual needs of different subgroups of OA patients and to set priorities for rehabilitation programs. ${ }^{29}$

Some limitations of this study need to be addressed. Although there has been an intervention (ie, the patients' arthroplasty), the data were analyzed in terms of a longitudinal observation study. There was no control group as the research question did not include an evaluation of the surgery itself. The HPA questionnaire used in this study has not been formally validated for assessment of PA in older populations and OA patients. However, the consistency of scores with the accelerometric data suggests that this tool is applicable in this setting. Another potential limitation to the study is that although participants were instructed not to share or discuss 
their questionnaire answers with their partners it cannot be ruled out that the participants exchanged some information. With regard to accelerometry, it should finally be noted that the use of a single device on the subjects' waist did not allow all activities to be recorded correctly. A weak point here is especially the recording of certain activities such as climbing stairs, riding a bicycle or walking on slopes, which so far can only be evaluated insufficiently in this setup. Finally, the investigated collective is small, so the results should be considered with caution.

\section{Conclusions}

Despite the stated limitations of this study, we believe it provides important information for surgeons, rehabilitation physicians and therapists. Current measures for the surgical treatment of OA show good results in eliminating pain and functional limitations of older patients. However, one year after surgery and rehabilitation, the habitual $\mathrm{PA}$ and $\mathrm{SB}$ of the patient and the supporting partner are exactly the same as before the intervention. The involved healthcare experts should counsel older patients and partners accordingly and recognize the demands placed on the patient's partners in their roles as caregivers for total joint arthroplasty patients. ${ }^{36}$ Future research should focus on the preoperative assessment of the partners' physical and psychical ability to care for the patients, and on perioperative programs that involve both partners as a unit.

\section{Data Sharing Statement}

Data will be made available upon reasonable request to the corresponding author.

\section{Ethics Approval and Informed Consent}

The study was conducted in agreement with the Declaration of Helsinki and was approved by the local Ethics Committee of the WWU in Münster, Germany (2017-613-f-S). All participants gave written informed consent.

\section{Acknowledgments}

We thank the patients and especially their partners for their willingness to participate in our study.

\section{Author Contributions}

All authors made a significant contribution to the work reported, whether that is in the conception, study design, execution, acquisition of data, analysis and interpretation, or in all these areas; took part in drafting, revising or reviewing the article; gave final approval of the manuscript to be published; and agree to be accountable for all aspects of the work.

\section{Funding}

This research did not receive any specific grant from funding agencies in the public, commercial, or not-forprofit sectors. We acknowledge support from the Open Access Publication Fund of the University of Muenster.

\section{Disclosure}

The authors declared no potential conflicts of interest with respect to the research, authorship, and/or publication of this article.

\section{References}

1. Wallis JA, Webster KE, Levinger P, Taylor NF. What proportion of people with hip and knee osteoarthritis meet physical activity guidelines? A systematic review and meta-analysis. Osteoarthritis Cartilage. 2013;21(11):1648-1659. doi:10.1016/j.joca.2013.08.003

2. Lee J, Chang RW, Ehrlich-Jones L, et al. Sedentary behavior and physical function: objective evidence from the osteoarthritis initiative. Arthritis Care Res (Hoboken). 2015;67(3):366-373. doi:10.1002/acr.22432

3. Piercy KL, Troiano RP. Physical activity guidelines for Americans from the US Department of Health and Human Services cardiovascular benefits and recommendations. Circ Cardiovasc Qual Outcomes. 2018;11(11):e005263. doi:10.1161/CIRCOUTCO MES.118.005263

4. Dunlop DD, Song J, Lee J, et al. Physical activity minimum threshold predicting improved function in adults with lower-extremity symptoms. Arthritis Care Res (Hoboken). 2017;69(4):475-483. doi:10.1002/acr.23181

5. White DK, Tudor-Locke C, Zhang Y, et al. Daily walking and the risk of incident functional limitation in knee osteoarthritis: an observational study. Arthritis Care Res (Hoboken). 2014;66(9):1328-1336. doi:10.1002/acr.22362

6. Master H, Thoma LM, Christiansen MB, Polakowski E, Schmitt LA, White DK. Minimum performance on clinical tests of physical function to predict walking 6000 steps/day in knee osteoarthritis: an observational study. Arthritis Care Res (Hoboken). 2018;70 (7):1005-1011. doi:10.1002/acr.23448

7. Hochberg MC, Altman RD, April KT, et al. American College of Rheumatology 2012 recommendations for the use of nonpharmacologic and pharmacologic therapies in osteoarthritis of the hand, hip, and knee. Arthritis Care Res. 2012;64(4):465-474. doi:10.1002/acr.21596

8. Kamaruzaman H, Kinghorn P, Oppong R. Cost-effectiveness of surgical interventions for the management of osteoarthritis: a systematic review of the literature. BMC Musculoskelet Disord. 2017;18(1):183. doi:10.1186/s12891-017-1540-2

9. Gonzalez Sáenz de Tejada M, Escobar A, Herrera C, García L, Aizpuru F, Sarasqueta C. Patient expectations and health-related quality of life outcomes following total joint replacement. Value Health. 2010;13(4):447-454. doi:10.1111/j.1524-4733.2009.00685.x

10. Harding P, Holland AE, Delany C, Hinman RS. Do activity levels increase after total hip and knee arthroplasty? Clin Orthop Relat Res. 2014;472(5):1502-1511. doi:10.1007/s11999-013-3427-3

11. Hammett T, Simonian A, Austin M, et al. Changes in physical activity after total hip or knee arthroplasty: a systematic review and meta-analysis of six- and twelve-month outcomes. Arthritis Care Res (Hoboken). 2018;70(6):892-901. doi:10.1002/acr.23415 
12. Mills K, Falchi B, Duckett C, Naylor J. Minimal change in physical activity after lower limb joint arthroplasty, but the outcome measure may be contributing to the problem: a systematic review and meta-analysis. Physiotherapy. 2019;105(1):35-45. doi:10.1016/j. physio.2018.04.003

13. Boltz M. The family caregiver: an untapped resource. Geriatr Nurs. 2012;33(2):137-139. doi:10.1016/j.gerinurse.2012.02.004

14. Martire LM, Stephens MAP, Mogle J, Schulz R, Brach J, Keefe FJ. Daily spousal influence on physical activity in knee osteoarthritis. Ann Behav Med. 2013;45(2):213-223. doi:10.1007/s12160-012-9442-x

15. Soto SH, Callahan LF, Bahorski S, et al. The role of cohabitating partner and relationship characteristics on physical activity among individuals with osteoarthritis. Int J Behav Med. 2019;26(5):522-530. doi:10.1007/s12529-019-09806-2

16. Khan CM, Iida M, Stephens MA, Fekete EM, Druley JA, Greene KA. Spousal support following knee surgery: roles of self-efficacy and perceived emotional responsiveness. Rehabil Psychol. 2009;54(1):28-32. doi:10.1037/a0014753

17. Stephens MA, Fekete EM, Franks MM, Rook KS, Druley JA, Greene K. Spouses' use of pressure and persuasion to promote osteoarthritis patients' medical adherence after orthopedic surgery. Health Psychol. 2009;28(1):48-55. doi:10.1037/a0012385

18. Pinquart M, Sorensen S. Spouses, adult children, and children-in-law as caregivers of older adults: a meta-analytic comparison. Psychol Aging. 2011;26(1):1-14. doi:10.1037/a0021863

19. Moellenbeck B, Horst F, Gosheger G, Theil C, Seeber L, Kalisch T. Does total hip or knee arthroplasty have an effect on the patients' functional or behavioral outcome and health-related quality of life of the affected partners? J Arthroplasty. 2020. doi:10.1016/j.arth.2020.08.066

20. Meyler D, Stimpson JP, Peek MK. Health concordance within couples: a systematic review. Soc Sci Med. 2007;64(11):2297-2310. doi:10.1016/j.socscimed.2007.02.007

21. Moellenbeck B, Kalisch T, Gosheger G, et al. Behavioral conformity of physical activity and sedentary behavior in older couples with one partner suffering from end-stage osteoarthritis. Clin Interv Aging. 2020;15:61-74. doi:10.2147/CIA.S222490

22. Moellenbeck B, Horst F, Gosheger G, Theil C, Seeber L, Kalisch T. Sedentary behavior in older patients before and after total hip arthroplasty: a prospective cohort study. Healthcare (Basel). 2020;8(3) doi:10.3390/healthcare8030346

23. Bernaards CM, Hildebrandt VH, Hendriksen IJ. Correlates of sedentary time in different age groups: results from a large cross sectional Dutch survey. BMC Public Health. 2016;16(1):1121. doi:10.1186/ s12889-016-3769-3

24. Altman RD. Criteria for classification of clinical osteoarthritis. J Rheumatol Suppl. 1991;27:10-12.

25. Migueles JH, Cadenas-Sanchez C, Ekelund U, et al. Accelerometer data collection and processing criteria to assess physical activity and other outcomes: a systematic review and practical considerations. Sports Med. 2017;47(9):1821-1845. doi:10.1007/s40279-017-0716-0

26. Choi L, Ward SC, Schnelle JF, Buchowski MS. Assessment of wear/ nonwear time classification algorithms for triaxial accelerometer Med Sci Sports Exerc. 2012;44(10):2009. doi:10.1249/ MSS.0b013e $318258 \mathrm{cb} 36$

Clinical Interventions in Aging

\section{Publish your work in this journal}

Clinical Interventions in Aging is an international, peer-reviewed journal focusing on evidence-based reports on the value or lack thereof of treatments intended to prevent or delay the onset of maladaptive correlates of aging in human beings. This journal is indexed on PubMed Central, MedLine, CAS, Scopus and the Elsevier
27. Kim Y, Barry VW, Kang M. Validation of the ActiGraph GT3X and activPAL accelerometers for the assessment of sedentary behavior. Meas Phys Educ Exerc Sci. 2015;19(3):125-137. doi:10.1080/ 1091367X.2015.1054390

28. Sasaki JE, John D, Freedson PS. Validation and comparison of ActiGraph activity monitors. J Sci Med Sport. 2011;14(5):411-416. doi:10.1016/j.jsams.2011.04.003

29. Sliepen M, Mauricio E, Lipperts M, Grimm B, Rosenbaum D. Objective assessment of physical activity and sedentary behaviour in knee osteoarthritis patients - beyond daily steps and total sedentary time. BMC Musculoskelet Disord. 2018;19(1):64. doi:10.1186/ s12891-018-1980-3

30. Ludwig FJ, Melzer C, Grimmig H, Daalmann HH. Kulturelle adaptation des Lequesne-Index fur Huft- und Kniegelenkserkrankungen im deutschen Sprachraum. [Cross cultural adaptation of the lequesne algofunctional indices for German speaking patients with osteoarthritis of the hip and the knee]. Rehabilitation (Stuttg). 2002;41 (4):249-257. doi:10.1055/s-2002-33273

31. Wagner P, Singer R. Ein Fragebogen zur Erfassung der habituellen korperlichen Aktivitat verschiedener Bevolkerungsgruppen. [A questionnaire for the registration of the habitual physical activity of different groups of population]. Sportwissenschaft. 2003;33 (4):383-397.

32. Evans JD. Straightforward Statistics for the Behavioral Sciences. Thomson Brooks/Cole Publishing Co; 1996.

33. Bellettiere J, Carlson JA, Rosenberg D, et al. Gender and age differences in hourly and daily patterns of sedentary time in older adults living in retirement communities. PLoS One. 2015;10(8):e0136161. doi:10.1371/journal.pone.0136161

34. Pauly T, Keller J, Knoll N, et al. Moving in sync: hourly physical activity and sedentary behavior are synchronized in couples. Ann Behav Med. 2020;54(1):10-21. doi:10.1093/abm/kaz019

35. Benatti FB, Ried-Larsen M. The effects of breaking up prolonged sitting time: a review of experimental studies. Med Sci Sports Exerc. 2015;47(10):2053-2061. doi:10.1249/MSS.0000000000000654

36. Kunkel ST, Sabatino MJ, Torchia MT, Jevsevar DS, Moschetti WE. Does the impact of joint arthroplasty extend beyond the patient? The effect of total joint arthroplasty on patient's significant others. J Arthroplasty. 2020;35(6):S129-s132. doi:10.1016/j.arth.2020.01.028

37. Adelman RD, Tmanova LL, Delgado D, Dion S, Lachs MS Caregiver burden: a clinical review. JAMA. 2014;311 (10):1052-1060. doi:10.1001/jama.2014.304

38. Shephard RJ. Limits to the measurement of habitual physical activity by questionnaires. Br J Sports Med. 2003;37(3):197-206; discussion 206. doi:10.1136/bjsm.37.3.197

39. Harris TJ, Owen CG, Victor CR, Adams R, Ekelund U, Cook DG. A comparison of questionnaire, accelerometer, and pedometer: measures in older people. Med Sci Sports Exerc. 2009;41(7):1392-1402. doi:10.1249/MSS.0b013e31819b3533

40. Chow W. An investigation of carers' burden: before and after a total hip replacement. Br J Occup Ther. 2001;64:503e8.
Bibliographic databases. The manuscript management system is completely online and includes a very quick and fair peer-review system, which is all easy to use. Visit http://www.dovepress.com/ testimonials.php to read real quotes from published authors. 